\title{
Microcorrente no processo de cicatrização: revisão da literatura
}

\author{
The microcurrent in the wound healing process: literature review
}

\author{
Microcorriente en el proceso de curación: revisión de la literatura \\ Anderson MARTELLI ${ }^{1}$ \\ Viviane THEODORO ${ }^{2}$ \\ Verena Eduarda ZANIBONI ${ }^{3}$ \\ Bruna Alves de FREITAS ${ }^{3}$ \\ Gabriela Maiara PASTRE ${ }^{4}$ \\ Karina Marques de MELO 5 \\ Thiago Antonio Moretti de ANDRADE ${ }^{6}$ \\ Gláucia Maria Tech dos SANTOS ${ }^{7}$ \\ ${ }^{I}$ Mestrando do Programa de Pós Graduação em Ciências Biomédicas - Centro Universitário Hermínio Ometto, FHO - UNIARARAS, Araras-SP \\ Especialista em Laboratório Clínico pela Faculdade de Ciências Médicas - UNICAMP, Campinas-SP \\ ${ }^{2}$ Mestranda do Programa de Pós Graduação em Ciências Biomédicas - Centro Universitário Hermínio Ometto, FHO - UNIARARAS, Araras-SP \\ ${ }^{3}$ Graduanda em Fisioterapia pelo Centro Universitário Hermínio Ometto, FHO - UNIARARAS, Araras-SP \\ ${ }^{4}$ Graduanda em Farmácia pelo Centro Universitário Hermínio Ometto, FHO - UNIARARAS, Araras-SP \\ ${ }^{5}$ Graduanda em Biomedicina pelo Centro Universitário Hermínio Ometto, FHO - UNIARARAS, Araras-SP \\ ${ }^{6}$ Professor Assistente Doutor, Programa de Pós Graduação em Ciências Biomédicas, \\ Centro Universitário Hermínio Ometto, FHO - UNIARARAS, Araras-SP \\ ${ }^{7}$ Professor Assistente Doutor, Programa de Pós Graduação em Ciências Biomédicas, \\ Centro Universitário Hermínio Ometto, FHO - UNIARARAS, Araras-SP
}

\begin{abstract}
Resumo
A cicatrização de feridas é uma resposta biológica à lesão tecidual sendo que diversos métodos terapêuticos estão sendo desenvolvidos e empregados para acelerar tal processo. Entre esses se observa a utilização de estimulação elétrica de baixa intensidade que favorece numerosos eventos no processo cicatricial assim como estimula eventos celulares, a circulação local e também promove alívio de quadros dolorosos. Sua aplicação é subsensorial não causando dor e/ou desconforto. Efeitos colaterais não são observados constituindo uma técnica de baixo custo e não invasiva. O presente estudo descreve a utilização da microcorrente (MC) como método não farmacológico no processo de reparo tecidual. Para isso foi realizada revisão da literatura, sendo consultados artigos científicos localizados nas bases de dados Medline, Scielo, Lilacs, portal de Periódicos CAPES e Google Acadêmico, publicados entre os anos de 1997 e 2014. Conclui-se que a MC é uma técnica inovadora e eficiente, podendo ser usada de forma isolada ou associada a outros métodos físicos ou a curativos visando à reparação tecidual, diminuição da inflamação, dor, podendo ser utilizada na melhoria da qualidade de vida.
\end{abstract}

Descritores: Condutas Terapêuticas; Terapia por Estimulação Elétrica; Cicatrização.

\begin{abstract}
Wound healing is a biological response to tissue injury and the different therapeutic methods are being developed and employed to accelerate this process. Among these methods, it has been observed the use of electrical stimulation of low intensity which promotes numerous events in the healing process as well as stimulates cellular events, the local circulation and also, promotes relief from painful conditions. Its application is subsensorial not causing pain and/or discomfort. Side effects are not observed constituting a low-cost and non-invasive technique. This study describes the use of microcurrent (MC) as a non-pharmacological method in the tissue repair process. This literature review was performed through consulting scientific articles searched in Medline, Scielo, Lilacs, portal CAPES Journals and Google Scholar, published between 1997 and 2014. The results point out the MC as an innovative and efficient technique, may be used alone or associated to other physical methods or curative in order to tissue repair, decrease inflammation, pain, and may be used in improving the quality of life.
\end{abstract}

Descriptors: Therapeutic Approaches; Electric Stimulation Therapy; Wound Healing.

\section{Resumen}

La cicatrización de heridas es una respuesta biológica a la lesión tisular y los diferentes métodos terapéuticos se están desarrollando y se emplea para acelerar este proceso. Entre ellas se observa el uso de la estimulación eléctrica de baja intensidad que promueve numerosos eventos en el proceso de curación, así como estimula los procesos celulares, la circulación local y también promueve el alivio de condiciones dolorosas. Su aplicación es subsensorial no causar dolor y / o incomodidad. No se observaron efectos secundarios que constituye un bajo costo y una técnica no invasiva. Este estudio describe el uso de microcorriente (MC) como un método no farmacológico en el proceso de reparación de tejidos. Por esta revisión de la literatura se llevó a cabo de la literatura, consultado con los artículos científicos que se encuentran en Medline, Scielo, Lilacs, portal de Revistas CAPES y Google Scholar, publicada entre 1997 y 2014. Se deduce que la MC es una técnica innovadora y eficiente puede ser utilizado solo o asociado con otros métodos físicos o fin curativo para la reparación de tejidos, disminuir la inflamación, el dolor, y puede ser utilizado en la mejora de la calidad de vida.

Descriptores: Conductas Terapéuticas; Terapia por Estimulación Eléctrica; Cicatrización de Heridas. 


\section{INTRODUÇÃO}

A pele é o maior órgão do corpo humano, respondendo por aproximadamente $16 \%$ do peso corporal $^{1,2}$, sendo considerada como a primeira barreira de proteção do organismo contra agentes externos e patógenos e por isso está sujeita a constantes acometimentos, assim, sua capacidade de regeneração torna-se muito importante para a homeostasia tecidual ${ }^{3}$. A camada mais externa da pele é formada por tecido epitelial do tipo pavimentoso estratificado queratinizado, que constitui a epiderme e abaixo desta, encontramos um tecido conjuntivo denominado derme. O tecido epitelial apresenta uma grande coesão entre suas células, mas é sensível à solução de continuidade precipitada por diversos fatores, expondo o tecido conjuntivo subjacente ${ }^{4}$. Entretanto, este importante órgão está sujeito a diferentes tipos de estímulos danosos, os quais desencadeiam e/ou acionam vias biológicas na tentativa de restabelecer a homeostasia desta estrutura. O conjunto dessas vias de reestabelecimento de funções recebe a denominação de cicatrização ${ }^{2}$.

As feridas são eventos que podem afetar a fisiologia da pele, em especial aquelas que acometem a camada dérmica ${ }^{2}$. As tentativas humanas de intervir no processo de cicatrização das feridas, acidentais ou provocadas intencionalmente como parte da realização de procedimentos, remontam à antiguidade, demonstrando que desde então já se reconhecia a importância de protegê-las de forma a evitar que se complicassem e repercutissem em danos locais ou gerais para o paciente 5 .

Atualmente estamos vivenciando grandes avanços na compreensão dos processos e fenômenos envolvidos na regeneração tecidual, mas embora ocorram grandes investimentos nessa área, é alta a incidência e prevalência de feridas crônicas, com consequências sociais significativas sobre os portadores que podem desenvolver sequelas crônicas, absteísmo do trabalho e de suas atividades normais ${ }^{5}$. Além disso, os custos dos tratamentos de patologias relacionadas à deficiência cicatricial aumentam a importância desses estudos em busca de medicamentos e curativos capazes de interagir com o tecido lesado, tendo por objetivo acelerar o processo regenerativo local.

O retardo de cicatrização como ocorre no caso de úlceras de pés diabéticos, constitui um grave problema mundial, tanto financeiro quanto social ${ }^{6} \mathrm{e}$ por isso, se faz necessário o conhecimento a respeito de tal processo, para que se possa nele intervir, tornando-o mais eficaz e rápido, promovendo a homeostasia do organismo e o bem estar do paciente $^{7,8}$. Quando ocorre perda tecidual, esta pode atingir completa ou incompletamente a derme, ou mesmo atingir todo o órgão, chegando ao tecido celular subcutâneo determinando os tipos de feridas. A cicatrização também depende de vários fatores, locais e gerais, como: localização anatômica, tipo da pele, raça, técnica cirúrgica utilizada dentre outros ${ }^{5}$.

O mecanismo de cicatrização é um complexo processo biológico que envolve uma cascata perfeita e coordenada de células e eventos moleculares para a reconstituição de tecidos lesionados ${ }^{6,10}$. Este processo surge como resposta do tecido a lesões induzidas por trauma ou por procedimentos cirúrgicos sendo caracterizado por três fases que se sobrepõem e apresentam característica especificas: fase inflamatória, fase proliferativa e fase de remodelação ${ }^{2}$.

Durante a fase inflamatória, ocorre hemostasia, migração de leucócitos e início da cascata de reparação tecidual. A fase proliferativa caracteriza-se pela fibroplasia, angiogênese e reepitelização e na fase de remodelação, o colágeno, principal componente da derme, sofre "turnover" ocorrendo mudança do tipo de colágeno que compõe a matriz extracelular: o colágeno tipo III é inicialmente mais abundante que o tipo I e, ao longo deste processo, este vai sendo degradado ativamente enquanto que o colágeno I tem sua produção aumentada pelos fibroblastos ${ }^{2}$.

Apesar de alguns avanços recentes na compreensão destes processos básicos, os distúrbios de cicatrização de feridas continuam a provocar doenças e até mesmo a morte de pessoas ao redor do mundo ${ }^{9,11}$.

Segundo Freitas et al. ${ }^{12}$ o tratamento de feridas busca o fechamento rápido da lesão de forma a se obter cicatriz funcional e esteticamente satisfatória. Agentes físicos como o laser e microcorrente (MC) apresentam efeitos positivos sobre a aceleração do processo cicatricial atuando na sequência de eventos fisiológicos e bioquímicos como a inflamação, síntese de colágeno, formação do tecido de granulação e reepitelização $0^{13}$. Na prática clínica dos fisioterapeutas, a utilização destas terapias como forma de potencialização dos efeitos acima mencionados tem sido observada com frequência ${ }^{12}$.

Em relação à $\mathrm{MC}$, as funções fisiológicas dos tecidos biológicos são intermediadas por correntes elétricas endógenas e que possuem intensidade na faixa de microamperes $(\mu \mathrm{A})$. Assim, estimulações elétricas exógenas teriam maiores efeitos se fossem realizadas com intensidades semelhantes às das correntes endógenas. A terapia com estimulação por MC pode, então, ser vista como catalisadora nos processos iniciais e de sustentação em numerosas reações químicas e elétricas que ocorrem no processo cicatricial $^{14}$. Atualmente verifica-se um grande número de pesquisas com $\mathrm{MC}$ voltadas para conhecimento e melhora do tratamento de feridas ${ }^{15}$ sendo que a cicatrização de uma ferida é medida em 
parte por sinais elétricos ${ }^{16}$. Portanto, a exposição artificial de feridas à estimulação elétrica altera o processo de cicatrização/reparo.

Considerando que as feridas podem promover alterações funcionais importantes decorrentes do quadro doloroso, que muitas vezes pode afastar pessoas em idade produtiva do trabalho, agravando a situação socioeconômica e dificultando o acesso até mesmo ao tratamento pela dificuldade de locomoção, e tendo em vista a cicatrização como um processo complexo, o presente estudo tem como objetivo realizar revisão sistemática da literatura especializada abordando a utilização da MC como método não farmacológico no processo de reparo tissular.

\section{MATERIAL E MÉTODO}

Para a composição da presente revisão foi realizado um levantamento bibliográfico nas bases de dados Medline, Scielo, Lilacs, Portal de Periódicos da Coordenação de Aperfeiçoamento de Pessoal de Nível Superior (CAPES) e busca de dados no Google Acadêmico de artigos científicos publicados até 2014 utilizando como descritores em português e inglês, isolados ou em combinação: microcorrente; cicatrização; reparo tecidual; feridas. Adicionalmente realizou-se consulta de livros acadêmicos para complementação das informações sobre a utilização da MC no reparo tecidual.

Para seleção do material foi obedecido fluxograma compreendendo três etapas (Figura 1).

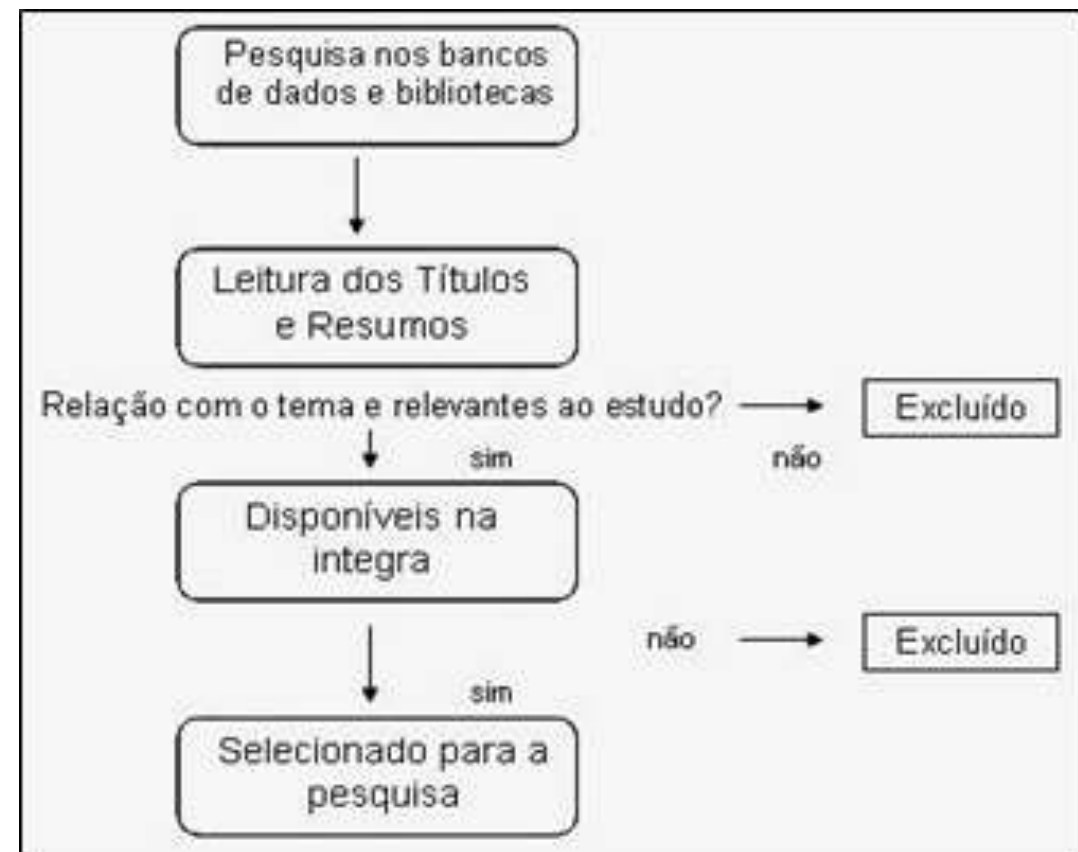

Figura 1. Fluxograma relativo às etapas de seleção dos artigos utilizados na pesquisa

A primeira foi caracterizada pela pesquisa do material que compreendeu entre os meses de Dezembro de 2014 a novembro de 2015 com a seleção de 38 trabalhos. A segunda compreendeu a leitura dos títulos e resumos dos trabalhos, visando uma maior aproximação e conhecimento, sendo excluídos os que não tivessem relação e relevância com o tema. Após essa seleção, buscaram-se os textos que se encontravam disponíveis na íntegra, totalizando 30 trabalhos, sendo estes, inclusos na revisão.

Dos artigos selecionados e incluídos na pesquisa constituíram ensaios clínicos, artigos originais, revisões e revisões sistemáticas da literatura. Como critérios de elegibilidade e inclusão dos artigos, analisaram-se a procedência da revista e indexação, estudos que apresentassem dados referentes à utilização da MC no processo de cicatrização publicados entre os anos de 1997 até o mais atual 2014. Na leitura e avaliação, os artigos que apresentaram os critérios de elegibilidade foram selecionados e incluídos na pesquisa por consenso.

Como critério de exclusão utilizou-se referência incompleta e informações presentemente desacreditadas, já que essa pesquisa visa revisar conhecimentos atualizados sobre o tema.

\section{CICATRIZAÇÃO E MICROCORRENTE}

Cicatrização é o processo pelo qual um tecido lesado é substituído por tecido conjuntivo vascularizado, quer a lesão tenha sido traumática ou necrótica ${ }^{17}$. Tal processo pode ocorrer espontaneamente por primeira intenção quando há união imediata das bordas da ferida, evolução asséptica e cicatriz linear ou por segunda intenção, quando a lesão apresenta-se extensa e infectada, com grande destruição tecidual e as bordas não se mantém unidas $^{18}$.

Logo após a ocorrência de um ferimento, um conjunto de eventos bioquímicos se estabelece para reparar o dano tecidual ${ }^{19}$. Como consequência, o sangue preenche a área lesada, seguido da coagulação, desidratação e formação de crosta, protegendo a lesão. Com a liberação de mediadores químicos por parte do coágulo, desenvolve-se uma reação inflamatória aguda ${ }^{4}$. Em decorrência do próprio trauma ou da ativação celular, o microambiente tem sua composição físico-química alterada (baixa tensão de $\mathrm{O}_{2}$, diminuição de $\mathrm{pH}$, presença de espécies reativas de nitrogênio e oxigênio) sendo esta, uma outra forma de sinalização que ativa as células envolvidas no reparo tecidual $^{20}$.

Atualmente a MC, corrente elétrica de baixa intensidade, atua sobre os tecidos em nível celular restaurando a bioeletricidade. Sua aplicação é subsensorial não estando associada à sensação desconfortável como outras correntes elétricas, ou seja, é indolor, além de não apresentar efeitos colaterais, ser de baixo custo e de fácil aplicação ${ }^{16}$. Assim, esta terapia representa um excelente instrumento para promover vascularização durante o processo de cicatrização ${ }^{12}$.

Kloth $^{21}$, em revisão da literatura, retrata que desde a década de 1960, muitas pesquisas têm sido 
realizadas com o objetivo de avaliar os efeitos da MC na cicatrização de feridas crónicas, uma vez que o número de publicações e ensaios clínicos de sucesso tem aumentado sensivelmente durante as últimas três décadas. $\mathrm{O}$ autor retrata sobre mudanças nas células epiteliais e na sua distribuição induzidas por campos elétricos constantes com atração de células pelo anodo (polo positivo) e catodo (polo negativo), onde os leucócitos migram para o anodo em correntes baixas e os macrófagos migram tanto para o catodo como para o anodo dependendo da força do campo.

O recurso eletroterapêutico desencadeia uma série de eventos, entre os quais, observa-se a proliferação fibroblástica, promovendo o aumento da síntese de colágeno, ações na contratilidade do tecido, neovascularização, incremento da síntese proteica, aumento da permeabilidade das membranas celulares e normalização da bioeletricidade tecidual ${ }^{12,16}$. Segundo os autores, este tipo de corrente pode ser vista como catalisadora nos processos iniciais e de sustentação em numerosas reações químicas e elétricas que ocorrem no processo cicatricial, acelerando em até $500 \%$ a produção do trifosfato de adenosina (ATP), sendo essa molécula a grande responsável pela síntese proteica e regeneração tecidual devido a sua participação em todos os processos energéticos da célula.

Inúmeros estudos foram bem sucedidos na utilização da $\mathrm{MC}$ no processo cicatricial ${ }^{21}$ sugerindo o efeito benéfico da terapia que restabelece a bioeletricidade tecidual e aumenta a velocidade de contração e fechamento da ferida em processo de cicatrização por segunda intenção. Alves et al. ${ }^{22} \mathrm{em}$ um estudo de caso relata que uma ferida póstraumática de $77 \mathrm{~cm}^{2}$ localizada na região anterior da coxa do membro inferior direito, não infectada, com cicatrização por segunda intenção há cinco meses, recebeu 30 sessões de $\mathrm{MC}(100 \mathrm{MHz}$, intensidade $=80$ $\mu \mathrm{A})$, três vezes por semana em dias alternados durante uma hora. Após 10 sessões, a área da ferida reduziu para $54 \mathrm{~cm}^{2}$ (percentual de redução de 29,9\%). Após 20 sessões, a redução foi de $64,9 \%$ e após 30 sessões foi 92,2\%. Após as sessões de MC a ferida apresentava-se mais hiperemiada, sugerindo o aumento do fluxo sanguíneo local, e que as bordas da ferida estavam contraindo, demonstrando o tensionamento tecidual.

Estudo clínico realizado por Rodopiano et al. ${ }^{16}$ em úlceras venosas de 10 pacientes de ambos os gêneros submetidos a 15 sessões com MC $(0,5 \mathrm{~Hz}$ e $250 \mu \mathrm{A} / 30 \mathrm{~min}$ ), duas vezes por semana mostrou um percentual de redução da área das lesões de 56,23\%. Este resultado comprova que a estimulação com MC neste tipo de lesão favorece o reparo tecidual, por normalizar o fluxo endógeno das correntes de lesão que se encontram na mesma faixa das correntes elétricas exógenas. Sendo assim, a MC se define como uma eletroestimulação fisiológica, homeostática e normalizadora nesse processo. $\mathrm{O}$ mesmo foi observado em úlceras venosas por Korelo et al. ${ }^{23} \mathrm{em}$ ensaio clínico controlado, com indivíduos de ambos os sexos, sedentários, idade superior a 50 anos, utilizando MC (pulso monofásico retangular, com reversão de polaridade a cada 3 segundos, 5 Hertz e $500 \mu \mathrm{A}$ por 4 semanas).

Migliato et al. ${ }^{24}$ através de estudos histomorfométricos observaram com a aplicação da MC modo contínuo, com intensidade de $10 \mu \mathrm{A}$, frequência de $0,3 \mathrm{~Hz}$, durante 2 minutos em feridas incisionais em ratos Wistar (Rattus norvegicus) favoreceu o aumento da área de reparo e o número total de células. Após 6 e 10 dias da lesão, a área da ferida foi completamente reepitelizada com a observação da derme preenchida com tecido fibroso, fibras de colágeno reorganizadas e elementos de fibrilas compactados. Também Passarini Jr et al. ${ }^{25}$, estudando feridas incisionais em ratos machos Wistar, observaram uma reparação tecidual expressiva no grupo tratado com $\mathrm{MC}$, intensidade de $10 \mu \mathrm{A}$, por 2 minutos com aumento do número total de células, assim como, maior formação de novos vasos sanguineos. Mendonça et al. ${ }^{09}$ observaram a capacidade reparadora de $\mathrm{MC}$, intensidade de $10 \mu \mathrm{A}$, por 2 minutos associada ao gel de Aloe vera também em feridas incisionais em ratos e concluíram que a aplicação simultânea de ambos apresentou grande potencial de cicatrização no modelo experimental utilizado.

Freitas et al. ${ }^{12}$ estudando ratos Wistar (Rattus norvegicus), utilizaram MC e laser no tratamento de queimaduras de $2^{\circ}$ grau, por 10 dias, e observaram aumento da presença de fibroblastos na aplicação de laser ou MC, mas o mesmo não ocorreu quando se associaram as duas terapias. Os autores registraram uma melhora significativa no grupo tratado com MC em relação à presença de anexos cutâneos. Em relação ao aumento de fibroblastos e colágeno os resultados descritos corroboram com os resultados de Santos et al. $^{26}$, que usaram MC $(50 \mu \mathrm{A})$ no tratamento de queimaduras em ratos. Queimaduras de $3^{\circ}$ grau em pele de ratos foram tratadas com $\mathrm{MC}(80 \mu \mathrm{A}), 15$ minutos cada sessão, forma bipolar, e observou-se efeitos benéficos relacionados ao processo cicatricial, pois, após 21 dias da lesão houve redução da inflamação e aumento do número de vasos sanguíneos e fibroblastos ${ }^{27}$.

Úlceras crônicas e outros tipos de ferimentos comprometem a qualidade de vida, devido, principalmente, à dor e perda da independência, além da presença de infecção local. A MC, frequência de 5 
$\mathrm{Hz}$ e intensidade em $500 \mu \mathrm{A}$ foi eficaz na redução dos quadros álgicos, com apenas 4 semanas de intervenção nesses quadros ${ }^{23}$.

Com relação ao tempo de aplicação da $\mathrm{MC}$, Alves et al. ${ }^{22}$ relatam que não foram encontrados estudos demonstrando o tempo de utilização ideal para a cicatrização de feridas cutâneas, sendo algo que pode ser analisado em estudos futuros. Além da terapia realizada, é importante salientar que fatores extrínsecos ao tratamento, como o metabolismo do paciente, os cuidados dispensados à lesão e mesmo sua etiologia são aspectos responsáveis pelo sucesso do tratamento como um todo, visando à redução da lesão ${ }^{22}$. Mendonça et al. ${ }^{28}$ utilizaram a MC $(2 \mu \mathrm{A} / 3$ min, $2 \mu \mathrm{A} / 5 \min$ e $5 \mu \mathrm{A} / 3 \mathrm{~min}$ ) em fraturas ósseas induzidas na tíbia de ratos, e observaram que o grau de recuperação foi melhor para $5 \mu \mathrm{A} / 3 \mathrm{~min}$.

Em extensa revisão de literatura realizada por Thakral et al. ${ }^{29}$ e Ud-Din e Bayat ${ }^{30}$ observa-se que, apesar das variações no tipo de corrente, duração e dosagem da estimulação elérica, a maioria dos estudos mostram uma melhoria significativa na redução do tamanho da ferida com o uso de MC, bem como a melhora da perfusão local não sendo observados relatos de complicações ou efeitos adversos na literatura existente. Portanto, a terapia por MC é segura, fácil de usar e não invasiva.

Há várias questões que permanecem sem respostas, como a identificação de qual tipo de ferida responde melhor ao tratamento, frequência e duração das sessões para cada tipo de ferida sendo necessários mais ensaios clínicos e experimentais para melhor compreensão da dosagem ótima, tempo e o tipo e modalidade de estimulação elétrica ${ }^{29,30}$.

\section{CONCLUSÃO}

A cicatrização de ferimentos é um processo que está presente na rotina clínica dos profissionais de saúde, dentre eles médicos, enfermeiros, fisioterapeutas e veterinários. Por ser um evento local, esse processo abrange uma gama de fatores que precisam interagir entre si para que haja uma evolução de forma eficiente nesse processo.

A MC é uma técnica inovadora e eficiente e que pode ser usada de forma isolada ou associada a outros métodos físicos ou a curativos nos protocolos de tratamento visando à reparação tecidual, diminuição da inflamação, dor e favorecimento das atividades da vida diária e qualidade de vida.

\section{REFERÊNCIAS}

1. Junqueira LC, Carneiro J. Histologia básica. Rio de Janeiro: Guanabara Koogan; 2004.

2. Isaac C, Ladeira PRS, Rego FMP, Aldunate JCB, Ferreira MC. Processo de cura das feridas: cicatrização fisiológica. Rev Med (São Paulo). 2010;89(3/4):125-31.

3. Nogueira RMB, Kitamura EA, Aguiar OM. Estudo clínico da reparação tecidual de feridas cutâneas de cães tratados com papaína e colagenase. Nos. Clín. 2005;8(43):25-8.

4. Nitz AC, Ely JB, d'Acampora AJ, David RiveroTames DR, Corrêa BP. Estudo morfométrico no processo de cicatrização de feridascutâneas em ratos, usando: Coronopudidymus e Calendula officinali. Arq Catar Med. 2006:35(4):74-9.

5. Mandelbaum SH, Di Santis EP, Mandelbaum MHS. Cicatrização: conceitos atuais e recursos auxiliares - Parte 1. An Bras Dermatol. 2003;78(4):393-410.

6. Mendonça RJ, Coutinho-Netto J. Aspectos celulares da cicatrização. An Bras Dermatol. 2009;84(3):257-62.

7. Oliveira BVPM, Dias RVC. Cicatrização de feridas: fases e fatores de influência. Acta Veterinaria Brasilica. 2012;6(4):267-71.

8. Cavalcante LC, Moreira MC, Mota OML, Turatti E, Viana FAC, Pereira SLS. Efeito da pedra umes no processo de cicatrização tecidual. Estudo histológico em dorso de ratos. Braz J Periodontol. 2012;22(1):69-73.

9. Mendonça FAS, Passarini Junior JR, Esquisatto MAM, Mendonça JS, Franchini CC, Santos GM. Effects of the application of Aloe vera (L.) and microcurrent on the healing of wounds surgically induced in Wistar rats. Acta Cir. Bras 2009;24(2):150-5.

10. Lima ROL, Rabelo ER, Moura VMBD, Silva LAF, Tresvenzol LMF. Cicatrização de feridas cutâneas e métodos de avaliação. Revisão de literatura. Revista CFMV. 2012;56(2):53-9.

11. Branski RC, Rosen CA, Verdolini K, Hebda PA. Biochemical markers associated with acute vocal fold wound healing: a rabbit model. J Voice. 2005;19(2):283-9.

12. Freitas, RPA, Barcelos APM, Nóbrega BM, Macedo AB, Oliveira AR, Ramos AMO, Vieira WHB. Laserterapia e microcorrente na cicatrização de queimadura em ratos. Terapias associadas ou isoladas? Fisioter Pesq. 2013;20(1):24-30.

13. Meyerholz DK, Piester TL, Sokolich JC, Zamba GK, Light TD. Morphological parameters for assessment of burn severity in an acute burn injury rat model. Int J Exp Pathol. 2009;90(1):26-33.

14. Guirro E, Guirro R. Fisioterapia dermatofuncional. 3. ed. São Paulo: Manole; 2004.

15. Kitchen S. Bazin S. Eletroterapia de Clayton. 10. ed. Rio de Janeiro: Manole; 2004. 
16. Rodopiano, N., Chagas, F., Silva, S.A., Santos, M.C.B. Intervenção da fisioterapia dermatofuncional com microcorrente e drenagem linfática na úlcera venosa. Anais do $10^{\circ}$ Fórum Científico da Faculdade de Ciências Médicas da Paraíba; 2013; Campus I da Faculdade de Ciências Médicas da Paraíba-FCM/PB.

17. Panobianco MS, Sampaio BAL, Caetano EA, Inocenti A, Gozzo TO. Comparação da cicatrização pós-mastectomia entre mulheres portadoras e não-portadoras de diabetes mellitus. Rev Rene. 2010;11:15-22.

18. Becker PFL. Patologia Geral. São Paulo: Sarvier; 1997; 127-33.

19. Paganela JC, Ribas L.M, Santos CA, Feijó LS, Nogueira CEW, Fernandes CG. Abordagem clínica de feridas cutâneas em equinos. RPCV. 2009;104(569-572):13-18.

20. Balbino CA, Pereira LM, Curi R. Mecanismos envolvidos na cicatrização: uma revisão. Rev Bras. Ciênc Farm. 2005;41(1):27-51.

21. Kloth LC. Electrical stimulation for wound healing: a review of evidence from in vitro studies, animal experiments, and clinical trials. Int $\mathbf{J}$ Low Extrem Wounds. 2005;4(1);23-44.

22. Alves, GPB, Leal CT, Carneiro R.A, Alves R, Resende TR. Utilização da microcorrentes no processo de cicatrizaçãode feridas em paciente pós-traumático: estudo de caso. In: Congresso Brasileiro de Fisioterapia Dermato Funcional; 2012, Recife.

23. Korelo RIG, Valderramas S, Ternoski B, Medeiros DS, Andres LF, Adolph SMM. Aplicação da microcorrente como recurso para tratamento de úlceras venosas: um estudo piloto Rev Latino-Am. Enfermagem. 2012;20(4):(08 telas).

24. Migliato KF, Chiosini M A, Mendonça FAZ, Esquisatto MAM, Salgado HR, Santos GMT. Effect of Glycolic Extract of Dillenia indica L. combined with Microcurrent Stimulation on Experimental Lesions in Wistar Rats. WOUNDS 2011;23(5):111-20.

25. Passarini Junior JR, Gaspi FOG, Neves LMG, Esquisatto MAM, Santos GMT, Mendonça FAS. Application of Jatrophacurcas L. seed oil (Euphorbiaceae) and microcurrent on the healing of experimental wounds in Wistar rats. Acta Cir Bras.2012;27(7):441-7.

26. Santos VNS, Ferreira LM, Horibe EK, Duarte IS. Electric microcurrent in the restoration of the skin undergone a trichloroacetic acid peeling in rats. Acta Cir Bras. 2004;19(5):466-70.

27. Steffani JA, Kroth A, Lorencete NA, D’Agostini FM. Uso de microcorrentesna cicatrização tecidual Evidência (UNOESC). 2011;11(1):43-50.
28. Mendonça FAS, Santos GMT, Esquisatto MAM, Alves AA, Passos LE, Mendonça JS. Efeito da aplicação de microcorrentes na osteogênese após fratura. RGO, 2005;53(3):193-197.

29. Thakral G, LaFontaine J, Najafi B, Talal TK, Kim P, Lavery LA. Electrical stimulation to accelerate wound healing. Diabet Foot Ankle. 2013; 4: 10.3402/dfa.v4i0.22081.

30. Ud-Din S, Bayat A. Electrical Stimulation and Cutaneous Wound Healing: A Review of Clinical Evidence. Healthcare. 2014;2(4):445-67; doi:10.3390/healthcare2040445.

\section{CONFLITO DE INTERESSES}

Os autores declaram não haver conflitos de interesse.

\section{AUTOR PARA CORRESPONDÊNCIA}

Anderson Martelli

martellibio@hotmail.com 